A. ARAI

KODAI MATH. J.

9 (1986), 161-164

\title{
ON UNIQUENESS THEOREM CONCERNING THE RENORMALIZED SCHWINGER-DYSON EQUATIONS OF FIRST ORDER
}

\author{
-A remark on the preceding paper by A. Inoue
}

\author{
By Asao Arai
}

In the preceding paper [1]. Inoue considered some Schwinger-Dyson (SD) equations of first order requiring "renormalizations": he derived the renormalized $\mathrm{SD}$-equation and constructed an explicit solution for each case. But the problem of the uniqueness is left open there.

Here we give an affirmative answer to that problem. The point of our method lies in a reduction of (SD)-equations to partial differential equations with finite numbers of variables of a certain type; it is essential that the order of the (SD)-equations is one.

For simplicity, we consider only the simplest one in [2]. The method can be easily extended to other cases. We shall carry over the notation in the preceding paper unless otherwise stated.

We first define a class of fundamental solutions of the d'Alembertian $\square$ :

Definition. Let $G$ be a distribution on $\boldsymbol{R}^{4} \times \boldsymbol{R}^{4}$ satisfying

$$
\square x G(x, y)=\delta(x-y)
$$

( $G$ is called a fundamental solution of $\square$.) Then $G$ is said to be in $\boldsymbol{F}$ if and only if it is a tempered distribution on $\boldsymbol{R}^{4} \times \boldsymbol{R}^{4}$ such that, for all $u \in S\left(\boldsymbol{R}^{4}\right)$,

$$
(G u)(0, t)=\lim _{\varepsilon \rightarrow 0}\left\langle\rho_{\varepsilon},(G u)(\cdot, t)\right\rangle
$$

exists in $H^{-1}(\boldsymbol{R})$

Remark. The class $\boldsymbol{F}$ is rather "large".

We consider the following renormalized SD-equation for functionals $Z=$ $Z(p, u)$ on $S(\boldsymbol{R}) \times S\left(\boldsymbol{R}^{4}\right)$ :

$$
\left(\frac{d}{d t^{2}}+\omega_{0}^{2}-\frac{i \lambda^{2}}{4 \pi}\left|\frac{d}{d t}\right|\right) \frac{\delta Z}{\delta p(t)}=\frac{i}{\hbar} p(t) Z-\frac{i \lambda}{\hbar}(G u)(0, t) Z,
$$

Received September 19, 1985 


$$
\begin{aligned}
\square & \frac{\delta Z}{\delta u(x, t)}=\frac{l}{\hbar} u(x, t) Z-\lambda \delta(x) \frac{\delta Z}{\delta p(t)}, \\
& Z(0,0)=1 .
\end{aligned}
$$

Remark. In [1], $G$ is chosen as $G_{F^{-}}^{B}$. Here

$$
G_{F_{ \pm} \pm}^{B}((x, \hat{i}),(y, s))=\lim _{\varepsilon \rightarrow 0}(2 \pi)^{-4} \int_{R \times R^{3}-\tau^{2}+|\xi|^{2} \pm \imath \varepsilon} \frac{e^{i \xi(x-y)-\imath \tau(t-i)}}{d \tau d \xi,}
$$

which are in $\boldsymbol{F}$.

THEOREM. For every $G$ in $\boldsymbol{F}$, there exists at most one solution $Z=Z(p, u)$ of (SD)-equations (1)-(3) satisfying

where

$$
\delta Z / \delta p(t) \in H^{-1}(\boldsymbol{R}), \delta Z / \delta u(x, t) \in \boldsymbol{D}_{G},
$$

$$
\boldsymbol{D}_{G}=\left\{v \in S^{\prime}\left(\boldsymbol{R}^{4}\right) \mid\langle G u, \square v\rangle=\langle u, v\rangle \quad \text { for all } u \in S\left(\boldsymbol{R}^{4}\right)\right\} \text {. }
$$

Before proving the theorem, we prepare

LEMMA. Let $f(t, s)$ be in $C^{1}(\boldsymbol{R} \times \boldsymbol{R})$ satisfyng

$$
\begin{gathered}
\frac{\partial}{\partial t} f(t, s)=(a t+b s) f(t, s), \\
\frac{\partial}{\partial s} f(t, s)=(b t+c s) f(t, s), \\
f(0,0)=0 .
\end{gathered}
$$

for some constants $a, b$, and $c$. Then $f(t, s)=0$ for all $(t, s)$ in $\boldsymbol{R} \times \boldsymbol{R}$.

Proof. From (6) and (7), we get $f(0, s)=f(0,0) \exp \left(c s^{2} / 2\right)=0$ for all $s$ in $\boldsymbol{R}$. Combining this with (5), we have

$$
f(t, s)=\int_{0}^{t}\left(a t^{\prime}+b s\right) f\left(t^{\prime}, s\right) d t^{\prime} .
$$

Let $L>0$ be arbitrarily fixed and define a sequence $\left\{T_{n}\right\}_{n=1}^{\infty}$ by

$$
\begin{gathered}
T_{0}=0, \\
T_{n}=-1+\left[\left(T_{n-1}+1\right)^{2}+\frac{1}{M}\right]^{1 / 2}, \quad n \geqq 1,
\end{gathered}
$$

with

$$
M=\max \{|a|,|b| L\} .
$$

(Since the case $M=0$ is trivial, we assume $M>0$.) Then, we have from (8) that, for all $(t, s) \in\left[0, T_{1}\right] \times[-L, L] \equiv \Omega_{L, T_{1}}$, 


$$
|f(t, s)| \leqq M\left(T_{1}^{2} / 2+T_{1}\right)\|f\|_{L, T_{1}}=\frac{\|f\|_{L, T_{1}}}{2},
$$

where $\|f\|_{L, T_{1}}$ denotes the maximum of $|f|$ on $\Omega_{L, T_{1}}$. This implies that $f=0$ on $\Omega_{L, T_{1}}$. In the same manner, we can show successively that, for all $n \geqq 1$, $f=0$ on $\left[T_{n}, T_{n+1}\right] \times[-L, L]$. Therefore we conclude that $f=0$ on $[0, \infty) \times$ $[-L, L]$. Similarly, we have $f=0$ on $(-\infty, 0] \times[-L, L]$. Since $L$ is arbitrary, we get $f=0$ on $\boldsymbol{R} \times \boldsymbol{R}$. Q.E.D.

Proof of Theorem. Since equations (1)-(3) are linear in $Z$, we need only to show that any functional $Z_{0}$ satisfying (1), (2) and (4) with $Z_{0}(0,0)=0$ is identically zero. Let $(p, u)$ be any fixed element in $S(\boldsymbol{R}) \times S\left(\boldsymbol{R}^{4}\right)$ and put

$$
f(t, s)=Z_{0}(t p, s u), \quad t, s \in \boldsymbol{R} .
$$

Then, $f$ is in $C^{\prime}(\boldsymbol{R} \times \boldsymbol{R})$ and we have

$$
\begin{gathered}
\frac{\partial}{\partial t} f(t, s)=\left\langle p, \frac{\delta Z_{0}(t p, s u)}{\delta p}\right\rangle \\
\frac{\partial}{\partial s} f(t, s)=\left\langle u, \frac{\delta Z_{0}(t p, s u)}{\delta u}\right\rangle \\
f(0,0)=0 .
\end{gathered}
$$

Equations (1), (2) and condition (4) give equations (5) and (6) with

$$
\begin{aligned}
& a=\frac{\imath}{\hbar}\left\langle p,\left(A_{\lambda}^{R}\right)^{-1} p\right\rangle, \quad b=-\frac{i}{\hbar}\left\langle(G u)(0, \cdot),\left(A_{\lambda}^{R}\right)^{-1} p\right\rangle, \\
& c=\frac{i}{\hbar}\left[\langle u, G u\rangle+\lambda^{2}\left\langle(G u)(0, \cdot),\left(A_{\lambda}^{R}\right)^{-1} G(0, \cdot)\right\rangle\right] .
\end{aligned}
$$

By the above lemma, we have $f(t, s)=0, t, s \in \boldsymbol{R}$, which implies $Z_{0}(p, u)=0$.

Q.E.D.

Remarks. (a) The unique solution of (SD)-equations (1)-(3) satisfying (4) is given by that in [1] with $G$ in place of $G_{F^{-}}^{B}$.

(b) For any solution $Z$ of (SD)-equations (1)-(3) satisfying (4), the function $f(t, s)=Z(t p, s u)$ obeies equations (5) and (6) with (9), (10) and $f(0,0)=1$, as is seen from the above proof. A solution of those equations is easily found by simple computations as

$$
f(t, s)=e^{(1 / 2) a t^{2}+b t s+(1 / 2) c s^{2}} .
$$

By the above lemma, this is the unique solution. Therefore, we have proved that, any solution $Z$ of (SD)-equations (1)-(3) satisfying (4), if it exists, must be of the form

$$
Z(p, u)=e^{(1 / 2) a+b+(1 / 2) c} .
$$


with $a, b$ and $c$ given in (9) and (10). Thus, our method also gives a simple way of construction of solutions of SD-equations of first order.

Acknowledgement. The author would like to thank Prof. A. Inoue for comments.

\section{REFERENCES}

[1] A. INOUE, Some examples exhibiting the procedures of renormalization and gauge fixing-Schwinger-Dyson equations of first order, Kodai Math. J., to appear.

[2] A. InOuE, An explicit solution of a certain Schwinger-Dyson equation, Proc. Jap. Acad. Ser. A., 57 (1981), 477-480.

Department of Mathematics,

ToKyo Institute of TECHNOLOGY

Oh-okayama, Meguro-ku, Tokyo 152,

JAPAN 